\title{
Should I stop or should I go on? Disease modifying therapy after the first clinical episode of multiple sclerosis
}

\author{
Tobias Monschein $^{1}$ (1) - Sabine Salhofer-Polanyi ${ }^{1} \cdot$ Patrick Altmann $^{1} \cdot$ Tobias Zrzavy $^{1}$ - Assunta Dal-Bianco ${ }^{1}$. \\ Gabriel Bsteh ${ }^{1} \cdot$ Paulus Rommer $^{1} \cdot$ Thomas Berger $^{1} \cdot$ Fritz Leutmezer $^{1}$
}

Received: 1 June 2020 / Revised: 7 July 2020 / Accepted: 10 July 2020 / Published online: 14 September 2020

(c) The Author(s) 2020

\begin{abstract}
Introduction Treatment with disease-modifying therapies (DMT) in patients with clinically isolated syndrome (CIS) represents standard care in multiple sclerosis (MS) patients nowadays. Since a proportion of patients may show no evidence of disease activity (NEDA) after some time of treatment, the question might arise about the risks of stopping DMT.

Methods We present a cohort of 49 patients who started DMT immediately after CIS and had no evidence of disease activity (NEDA-3) for at least five years before discontinuation of therapy. Thereafter, patients underwent clinical and MRI followup for at least five consecutive years.

Results Of 49 patients discontinuing DMT, 53\% $(n=26)$ had NEDA for at least further five years, while $47 \%(n=23)$ showed either a relapse/disease progression $(18.4 \%, n=9)$, MRI activity $(14.3 \%, n=7)$ or both $(14.3 \%, n=7)$. The main predictive factor for sustained NEDA was age at DMT termination. Patients aged $>45$ years had a significantly lower risk of disease reactivation $(13 \%$ vs. $54 \%$ in patients aged $<45$ years, $p<0.001)$ after DMT discontinuation.

Discussion In CIS patients with immediate DMT after their first clinical episode, older age at the time of DMT discontinuation is the main predictive factor for sustained NEDA status.
\end{abstract}

\section{Introduction}

A clinically isolated syndrome (CIS) is defined as the first clinical symptom suggestive of inflammatory demyelination of the central nervous systems (CNS) without evidence for dissemination in time and space that are both necessary for a diagnosis of multiple sclerosis (MS) [1]. Natural history studies have shown that the majority of patients ultimately convert to clinically definite MS (CDMS) [2,3]. Younger age at onset, male sex, type of clinical symptoms at onset [2], presence of oligoclonal bands (OCB) in cerebrospinal fluid (CSF) [4], and magnetic resonance imaging (MRI) T2-lesion load [5] are the most important prognostic factors for this conversion. CIS and MS are diagnosed using the McDonald criteria, which were recently revised in 2017, resulting in increased sensitivity and consequently lower incidence of CIS [6].

Tobias Monschein

tobias.monschein@meduniwien.ac.at

1 Medizinische Universitat Wien, Wien, Austria
In randomized controlled trials, disease-modifying therapies (DMT) like interferon beta (IFN-beta) products and glatiramer acetate (GA) were shown to delay the conversion from CIS to CDMS significantly [7-10]. Consequently, IFN-beta and GA have been approved for CIS and are, thus, widely used in clinical routine [11].

However, prolonged periods of absence of disease activity may prompt consideration of DMT discontinuation in CIS patients and treating neurologists, especially if patients experience adverse events or syringe fatigue [12]. While the benefit-risk profiles of IFNb and GA are generally favourable, side effects, matters of convenience, and even economic burden, including costs for health care systems, may be considered in the discussion whether to continue or to stop DMT [13].

Robust data supporting the discontinuation of DMT are scarce to date, with a lack of evidence especially in clinically stable CIS patients. Natural history data suggest that inflammatory activity declines with age [14] and there is some evidence in CDMS from observational databases $[12,15,16]$ or unselected retrospective cohorts [17] with the limitation of considerable heterogeneity concerning both patient population as well as reasons for DMT discontinuation (ranging 
from pregnancy, lack of adherence, and side effects to stable disease course).

The primary goal of our study was to evaluate possible predictive factors for NEDA status after stopping DMT in a homogenous cohort of CIS patients, who started DMT after the very first clinical episode suggestive of MS and discontinued DMT after remaining free of disease activity for at least five years.

\section{Patients and methods}

This is a retrospective analysis of prospectively collected observational data. We recruited patients from the MS outpatient clinic of the Department of Neurology, Medical University of Vienna, diagnosed between 2001 and 2011 with a CIS according to McDonald criteria 2001 (see Fig. 1). The study was approved by the ethics committee of the Medical University of Vienna (EK 1203/2016).

Inclusion criteria were initiation of DMT (IFN-beta or GA) after a diagnosis of CIS was established. Even though the 2017 revision of the McDonald diagnostic criteria are the generally accepted criteria for MS diagnosis nowadays, for the purpose of this work, it is important to emphasize that we used the $2001 \mathrm{McD}$ onald diagnostic criteria (applicable when the majority of the patients from our cohort was diagnosed). This version defined CIS as a monosymptomatic event suggestive of a first inflammatory demyelinating event with an acute onset reaching a peak within 14 days in the absence of objective clinical evidence of a second lesion and without evidence for dissemination in time and space as derived from typical MRI findings and eventually from positive oligoclonal bands in the cerebrospinal fluid (CSF) [1]. The risk of misdiagnosis at this very early stage of the disease was minimized using the more conservative Barkhof MRI criteria in all patients, and CSF testing for oligoclonal bands (OCB). The Barkhof criteria consist of at least 1 gadolinium-enhancing lesion or at least 9 lesions on T2-weighted images, at least 3 periventricular lesions, at least 1 juxtacortical lesion and at least 1 infratentorial lesion. 3 of the 4 variables must be met [18].

After initiation of DMT, patients had to have no evidence of disease activity (NEDA-3) for at least five consecutive years before discontinuation of therapy [19]. In this case, the option of DMT discontinuation was discussed with the patient extensively; however, the decision was primarily based on the patient's individual choice. After discontinuation of DMT, patients underwent regular clinical examinations at least annually (clinical history with documentation of confirmed relapse and disease progression as measured by the Expanded Disability Status Scale (EDSS)) as well as annual MRI follow-ups for at least five consecutive years.

A relapse was defined as a typical symptom of an acute inflammatory demyelinating event lasting for at least $24 \mathrm{~h}$, at least 30 days apart from the last episode, and without associated temperature increase or recent infection.

Furthermore, a confirmed sustained EDSS increase of 0.5 or more at 6-month follow-up as compared to baseline (i.e. time of treatment initiation) was defined as EDSS progression. MRI activity was defined as either a new or enlarging $\mathrm{T} 2$ lesion or a new contrast-enhancing lesion as compared to a prior MRI. NEDA-3 was defined as the absence of relapse, disease progression and MRI activity [19].

Patients were divided into two groups: the first group was labelled "evidence of disease activity" (EDA) and included those patients who suffered either a relapse, EDSS progression, and/or MRI activity during the 5-year follow-up period; the second group was called "NEDA" comprising patients with no evidence of disease activity (NEDA-3) within five years of follow-up. In addition, various age cutoffs were analysed based on the median of the group (31y) and current available data with regard to the NEDA rate (40y and 45y) [12].

\section{Statistics}

The statistical evaluation of the collected data was done in SPSS (SPSS Inc. Version 26.0, Chicago, IL, USA). Continuous parametric variables were tested for normal distribution by Kolmogorov-Smirnov test. Categorical variables were expressed in frequencies and percentages, continuous parametric variables as either mean and $95 \%$ confidence intervals (95\% CIs) or median and range as appropriate depending on normal distribution. The primary endpoint of the study was the comparison of EDA versus NEDA after a minimum follow-up of at least five years.

Univariate differences between patient groups in categorical variables were evaluated using cross-tabulation and chisquare test corrected by Fisher's exact test. Numeric variables were analysed by independent $t$-test or Mann-Whitney $U$ test as appropriate depending on normal distribution.

Multivariate analyses were performed using binary logistic regression. Group comparisons regarding time to event (NEDA-3 status) were investigated using Kaplan-Meier curves and Cox regression models. The log-rank test was used for evaluation of significance. The significance level was set with a $p<0.05$ (two-sided).

\section{Results}

We included a total of 49 patients. The mean age at initial symptoms was $26.3 \pm 7.5$ years and $65 \%$ were female. Demographic and clinical characteristics are given in Tables 1 and 2 . Table 1 additionally shows our data in comparison to the 
baseline characteristics of the large phase III clinical CIS trials.

Demographic data among the various studies show comparable distributions. Although the proportion of monofocal and multifocal onset symptoms is distributed differently, the diagnostic certainty in our cohort is, however, supported by cerebrospinal fluid analyses in all our patients, demonstrating positive oligoclonal bands in $88 \%$ of patients.

$26(53 \%)$ of 49 patients presented with sustained NEDA at 5-year follow-up after having had discontinued DMT. In contrast, $47 \%(n=23)$ showed signs of clinical and/or MRI disease activity. Clinical (relapse and or disease progression) or MRI activity, or a combination of both was found in 18.4\% ( $n=9), 14.3 \%(n=7)$, and $14.3 \%(n=7)$, respectively.

Age at DMT discontinuation was significantly higher in the NEDA group $(p=0.003)$, which was the only factor with significant difference. However, there was also a tendency $(p=0.088)$ to a lower EDSS in the NEDA group (see Table 2). On the other hand, sex, type of DMT, presence or absence of OCB, CELs or spinal cord involvement on initial MRI, treatment duration, and CIS symptom $(p=0.774)$ showed no association with NEDA status.

In the multivariate analysis, by binary logistic regression, only age at discontinuation had a significant influence with higher age associated with higher probability of NEDA status. In the Cox regression as a function of time (until EDA or last follow-up), older age was significantly associated with lower risk of EDA, while female sex only showed a very modest association (Tables 3 and 4).
The mean time to risk of EDA was significantly shorter in patients younger than 31 years ( 26 months, $p=0.006$ ) compared to 40 years ( 31 months, $p=0.004$ ) and 45 years (37 months, $p=0.044$ ).

In a further step, time to EDA was compared according to different age cut-offs (31, 40 and 45 years) using the Kaplan-Meier function (see Figs. 1, 2, 3). Patients older than 40 years at DMT discontinuation had a significantly lower risk of recurrence of disease activity compared to those younger than 40 years (see Fig. $1 ; 18.8 \%$ vs. $60.6 \%$, $p=0.006$ ). A similar difference was found when comparing patients aged $>45 \mathrm{y} /<45 \mathrm{y}$ (see Fig. $2 ; 12.5 \%$ vs. $53.7 \%$, $\mathrm{p}=0.025$ ) and $>31 \mathrm{y} /<31 \mathrm{y}$ (see Fig. $3, p=0.003,28 \%$ vs. $66.7 \%)$.

\section{Discussion}

To our knowledge, this is the first study determining the risk of disease recurrence after DMT discontinuation in a cohort of patients, comparable to study populations of former phase III clinical trials in CIS, who started DMT immediately after CIS and remained NEDA-3 for at least 5 years thereafter.

In this cohort, older age at the time of DMT discontinuation was the main predictive factor for sustained NEDA after discontinuation. Patients aged $>45$ years had a significantly lower risk for disease reactivation (13\% vs. $54 \%, p<0.001)$ after DMT discontinuation. Furthermore, female gender was also shown to be a significant positive

Table 1 Comparison of demographical, clinical and paraclinical data in CIS studies (BENEFIT; CHAMPS; PRECISE; REFLEX) compared to this study

\begin{tabular}{|c|c|c|c|c|c|}
\hline & BENEFIT [20] & CHAMPS [21] & PRECISE [9] & REFLEX [10] & Own Cohort \\
\hline Treatment & SC IFNß-1b & IM IFNß-1a & GA & SC IFNß-1a & various \\
\hline Age (years) & 30 & 33 & 31 & 31 & 26 \\
\hline Female $(\%)$ & 71 & 73 & 65 & 67 & 65 \\
\hline HDMP for first demyelinating event (\%) & 71 & $\mathrm{nk}$ & 67 & 71 & 96 \\
\hline Monofocal symptoms (\%) & 52 & nk & nk & 56 & 92 \\
\hline NNO $(\%)$ & 29 & 49 & nk & nk & 33 \\
\hline Brainstem/Cerebellar (\%) & 22 & 30 & nk & nk & 18 \\
\hline Spinal (\%) & 34 & 21 & nk & nk & 5 \\
\hline Cerebral (\%) & 15 & nk & nk & nk & 44 \\
\hline Multifocal symptoms (\%) & 48 & $\mathrm{nk}$ & nk & nk & 8 \\
\hline EDSS & 1.5 & $\mathrm{nk}$ & 1.0 & nk & 1.0 \\
\hline Patients with CSF analysis (\%) & 68 & nk & nk & nk & 100 \\
\hline OCB positivity (\%) & 59 & nk & nk & nk & 88 \\
\hline Number of $\mathrm{T} 2$ lesions $\geq 9(\%)$ & 71 & $28(\geq 8)$ & 84 & 72 & 78 \\
\hline $\mathrm{CEL} \geq 1(\%)$ & 43 & 20 & 47 & 40 & 43 \\
\hline Time from onset to DMT start (days) & nk & 20 & 79 & nk & 83 \\
\hline
\end{tabular}

GA Glatirameracetate. IM IFN $\beta$-1a intramuscular Interferon beta-1a, SC IFN $\beta$-1b subcutaneous Interferon beta-1b, SC IFN $\beta$ - $1 a$ subcutaneous Interferon beta-1a, $H D M P$ high-dose methylprednisolone, $N N O$ neuritis nervi optici, EDSS expanded disability status scale, CSF cerebrospinal fluid, $O C B$ oligoclonal bands, $C E L$ contrast-enhancing lesion, $D M T$ disease-modifying therapy, $n k$ not known 
Table 2 Cohort characteristics

\begin{tabular}{|c|c|c|c|c|}
\hline & Total $(N=49)$ & $\begin{array}{l}\text { EDA } \\
(n=23)\end{array}$ & $\begin{array}{l}\text { NEDA } \\
(n=26)\end{array}$ & $\begin{array}{l}p \text {-value } \\
\text { (EDA vs NEDA) }\end{array}$ \\
\hline \multicolumn{5}{|l|}{ Age at (years) ${ }^{1}$} \\
\hline Initial symptoms & $26.3 \pm 7.4$ & $22.7 \pm 5.2$ & $29.5 \pm 7.7$ & $0.001^{4}$ \\
\hline DMT start & $26.5 \pm 7.5$ & $22.9 \pm 5.3$ & $29.8 \pm 7.8$ & $0.001^{4}$ \\
\hline DMT stop & $33.9 \pm 9.0$ & $30.9 \pm 7.0$ & $37.4 \pm 9.3$ & $0.003^{4}$ \\
\hline Female $^{2}$ & $32(65.3)$ & $14(60.9)$ & $18(69.2)$ & $0.564^{6}$ \\
\hline \multicolumn{5}{|l|}{$\mathrm{DMT}^{1}$} \\
\hline Time to start (months) & $2.7 \pm 1.9$ & $2.4 \pm 1.8$ & $3.0 \pm 1.9$ & $0.214^{4}$ \\
\hline Time on DMT (years) & $7.4(2.5)$ & $7.1 \pm 2.3$ & $7.6 \pm 2.6$ & $0.484^{4}$ \\
\hline \multicolumn{5}{|l|}{$\mathrm{EDSS}^{3}$} \\
\hline At DMT start & $1(0-3)$ & $1(0-2)$ & $1(0-3)$ & $0.730^{5}$ \\
\hline At DMT stop & $0(0-2)$ & $0(0-2)$ & $0(0-1)$ & $0.088^{5}$ \\
\hline OCB positivity $^{2}$ & $43(87.8)$ & $21(91.3)$ & $22(84.6)$ & $0.476^{6}$ \\
\hline \multicolumn{5}{|l|}{ Initial $\mathrm{MRI}^{2}$} \\
\hline CEL present & $21(42.9)$ & $10(43.5)$ & $11(42.3)$ & $0.934^{6}$ \\
\hline Spinal cord lesions & $21(42.9)$ & $10(43.5)$ & $11(42.3)$ & $0.934^{6}$ \\
\hline
\end{tabular}

${ }^{1}$ Mean and standard deviation

${ }^{2}$ Number and percentage

${ }^{3}$ Median and range. $p$-values calculated for comparison of EDA and NEDA group using

${ }^{4}$ Independent-test

${ }^{5}$ Mann-Whitney- $U$-test or

${ }^{6}$ Chi-square-test

$C E L$ contrast-enhancing lesion, $D M T$ disease-modifying therapy, EDA evidence of disease activity, $M R I$ magnetic resonance imaging, $N E D A$ no evidence of disease activity, $O C B$ oligoclonal bands

Table 3 Multivariate analysis by use of binary logistic regression regarding EDA versus NEDA; for therapy analysis GA was chosen as reference and compared to the different interferon products

\begin{tabular}{lccccccccr}
\hline Predictor & $B$ & SE & Wald $\chi^{2}$ & df & $p$ & OR & \multicolumn{2}{c}{ 95\% CI OR } \\
\cline { 6 - 9 } & & & & & & & & LL & UL \\
\hline Sex (female) & 1.258 & 0.851 & 2.184 & 1 & 0.139 & 3.518 & 0.664 & 18.650 \\
Age (stop) & -0.176 & 0.056 & 9.770 & 1 & 0.002 & 0.838 & 0.751 & 0.936 \\
OCB (positivity/ & 1.435 & 1.138 & 1.590 & 1 & 0.207 & 4.198 & 0.452 & 39.024 \\
negativity) & & & & & & & & \\
CEL (Yes/No) & -0.880 & 0.843 & 1.089 & 1 & 0.297 & 0.415 & 0.079 & 2.166 \\
Spinal MRI lesions (Yes/No) & 0.185 & 0.752 & 0.061 & 1 & 0.805 & 1.204 & .276 & 5.258 \\
Therapy (GA) & & & 0.691 & 3 & 0.875 & & & \\
IM IFNß-1a (30mcg) & 0.522 & 0.918 & 0.324 & 1 & 0.570 & 1.686 & 0.279 & 10.197 \\
SC IFNß-1b (250mcg) & -0.144 & 1.107 & 0.017 & 1 & 0.897 & 0.866 & 0.099 & 7.576 \\
SC IFNß-1a (44mcg) & -0.324 & 0.985 & 0.108 & 1 & 0.742 & 0.723 & 0.105 & 4.989 \\
\hline
\end{tabular}

$C E L$ contrast-enhancing lesion, GA Glatirameracetate, IM IFN $\beta$-1a intramuscular Interferon beta-1a, $S C$ $I F N \beta-1 b$ subcutaneous Interferon beta-1b, $S C$ IFN $\beta$ - 1 a subcutaneous Interferon beta-1a predictive factor for NEDA, although the small sample size $(n=49)$, level of significance $(p=0.047)$ and the lower limit of Hazard-Ratio (HR) (1.016) must be considered. All other variables (type of DMT, presence or absence of OCB, CELs, spinal cord involvement on initial MRI, treatment duration, and CIS symptom) did not show predictive relevance for disease reactivation after DMT discontinuation.
Our results are in line with large, multi-centre registrybased cohorts $[15,16]$ as well as single-centre cohorts $[12$, 17] of more heterogeneous patient groups, both with respect to the time of treatment initiation as well as the reason of DMT discontinuation.

In contrast to the above-mentioned studies, our cohort comprised only patients starting their DMT after clinical disease onset. Importantly, our cohort had a very low risk 
Table 4 Cox regression in dependence of time (until relapse or last follow-up) regarding EDA versus NEDA; for therapy analysis GA was chosen as reference and compared to the different interferon products

\begin{tabular}{lccccccccr}
\hline Predictor & $B$ & $S E$ & Wald $\chi^{2}$ & df & $p$ & & HR & \multicolumn{2}{c}{ 95\% CI HR } \\
\cline { 6 - 9 } & & & & & & & & LL & UL \\
\hline Sex (female) & 1.083 & .545 & 3.954 & 1 & 0.047 & 2.955 & 1.016 & 8.597 \\
Age (stop) & -0.164 & .042 & 15.010 & 1 & $<0.001$ & 0.849 & 0.781 & 00.922 \\
OCB (positivity/ & 0.676 & 0.824 & 0.673 & 1 & 0.412 & 1.967 & 0.391 & 9.894 \\
negativity) & & & & & & & & \\
CEL (Yes/No) & -0.404 & 0.497 & 0.661 & 1 & 0.416 & 0.668 & 0.252 & 1.769 \\
Spinal MRI lesions (Yes/No) & -0.077 & 0.505 & 0.023 & 1 & 0.0878 & 0.926 & 0.344 & 2.491 \\
Therapy (GA) & & & 1.482 & 3 & 0.686 & & & \\
IM IFNß-1a (30mcg) & 0.443 & 0.578 & 0.586 & 1 & 0.444 & 1.557 & 0.501 & 4.832 \\
SC IFNß-1b (250mcg) & 0.375 & 0.705 & 0.282 & 1 & 0.595 & 1.454 & 0.365 & 5.790 \\
SC IFNß-1a (44mcg) & -0.240 & 0.611 & .154 & 1 & 0.694 & 0.787 & 0.238 & 2.605 \\
\hline
\end{tabular}

$C E L$ contrast-enhancing lesion, GA Glatirameracetate, IM IFN $\beta$-1a intramuscular Interferon beta-1a, SC IFN $\beta-1 b$ subcutaneous Interferon beta- $1 \mathrm{~b}, S C$ IFN $\beta-1 a$ subcutaneous Interferon beta-1a

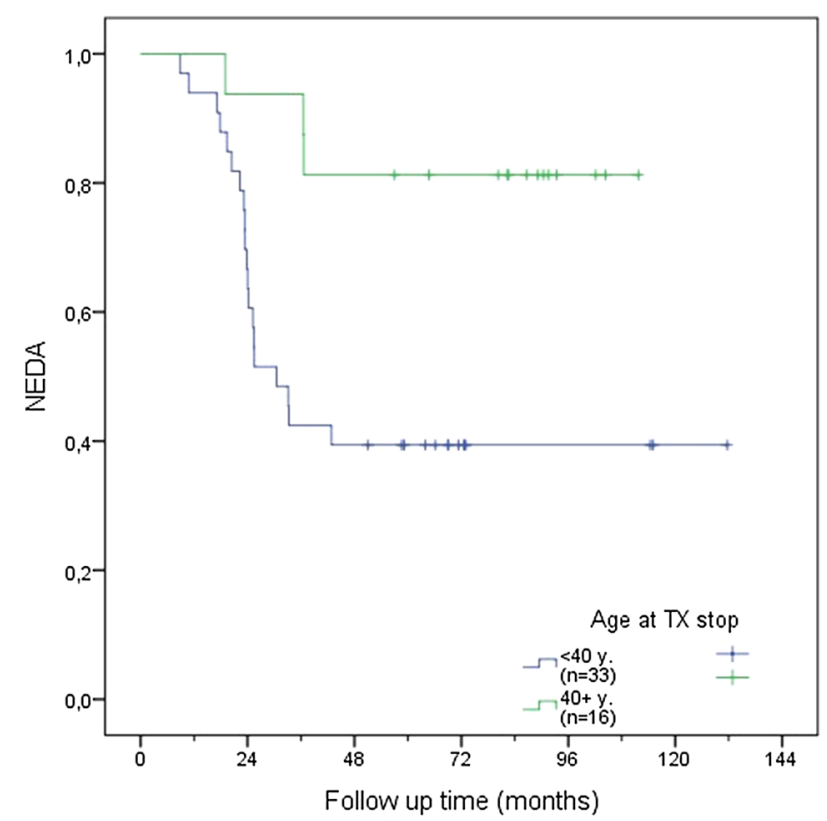

Fig. 1 NEDA rate according to age above or below age of 40 years at DMT discontinuation

of MS misdiagnosis due to application of Barkhof's strict MRI criteria in all participants as well as the presence of OCBs in CSF in the vast majority of patients (88\%). Moreover, the presence of positive OCB, CEL and spinal MRI lesions at the time of diagnosis was similarly distributed between NEDA and EDA patients, arguing against the possibility that NEDA patients may have had a higher risk of MS misdiagnosis.

In CDMS, Kister et al. found similar relapse rates in patients who stopped their DMT after a relapse-free period of at least 5 years as compared to those patients who remained on therapy [15]. However, the risk of post-DMT relapse was higher in younger patients, consistent with

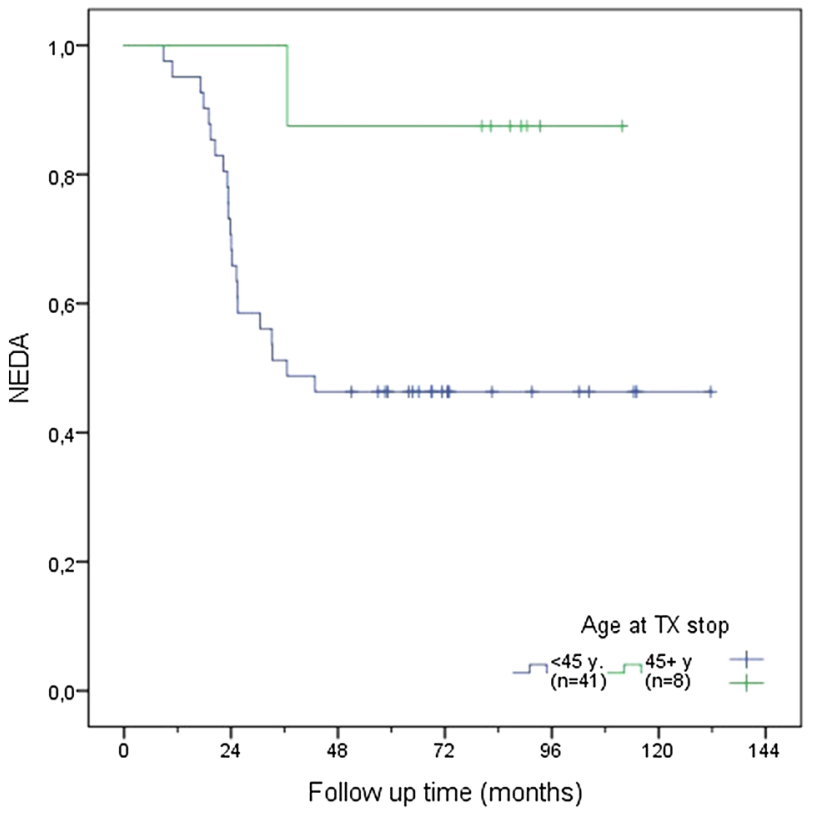

Fig. 2 NEDA rate according to age above or below age of 45 years at DMT discontinuation

natural history studies, showing a decline in relapse rate with age [22]. In contrast, the risk of disability progression in this cohort increased with age, again in accordance with natural history data [23].

Nevertheless, the risk of disability progression was higher in DMT stoppers as compared to patients with ongoing DMT use. This could be at least in part due to the fact that DMT discontinuation was more likely in older patients with a higher "natural" risk of conversion to SPMS.

From the same but extended cohort, Kister et al. [16] proposed a higher risk of post-DMT relapse in younger patients and females. This sex difference was vice versa 


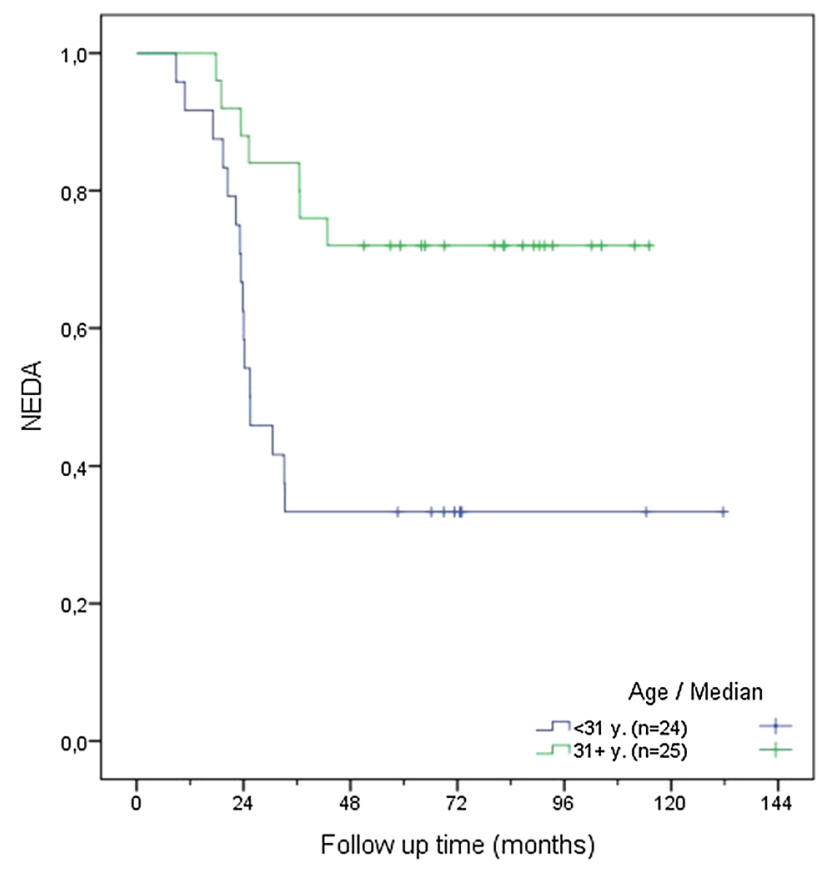

Fig. 3 NEDA rate according to age above or below the median age of the total group (31 years) at DMT discontinuation

in our cohort as already mentioned above. However, the effect sizes are very low rendering clinical relevance very unlikely. Similar results were also gained in a single-centre retrospective analysis of 221 prospectively followed RRMS patients who stopped DMT due to a variety of reasons [12]. Age $\geq 45$ years at discontinuation, absence of relapses for $\geq 4$ years on DMT before discontinuation, and absence of CELs on MRI were independent predictors of ongoing freedom of disease activity. Based on these data, we chose the cut-offs of 40 and 45 years in our analyses and cut off 45 showed the highest NEDA rate.

The strength of our study is the data completeness in terms of clinical and MRI data at baseline as well as during and post-DMT and the fact that DMT withdrawal was exclusively due to NEDA. It is important to point out that this is an essential difference to other studies where reasons for discontinuation were quite heterogenous $[12,15$, 16]. In contrast to these studies, who included patients with DMT initiation ranging from CIS to late-stage RRMS and even SPMS, we aimed to specifically address the question of DMT discontinuation in the increasing population of patients with DMT initiation after the very first clinical episode.

Especially in this patient group, both, patients and treating neurologists, might face the question "should we stop or go on with DMT?" Factors that heat up this discussion might be inconvenience, side effects, costs, but also the possibility of a favourable disease course, which was already reported in the pre-DMT era at least in a small proportion of patients [24]. Thus, not all patients might be in need of a long-term DMT without hampering long-term prognosis [25].

Our study has several limitations. First, sample size was small, and we do not have a control group. Furthermore, our patients were classified as CIS according to the McDonald criteria 2001. Considering that 43 of 49 patients had positive OCBs, and of those with negative OCBs, 5/6 had CELs on initial MRI, 48/49 patients would have been classified as definite MS according to the current McDonald criteria $[1,6]$.

In conclusion, in a homogenous group of CIS patients who started DMT immediately after the first clinical event and discontinued after achieving at least 5 years of NEDA, we could identify age at DMT discontinuation as the main predictive factor for re-occurrence of disease activity. These data should not encourage CIS patients to generally stop DMT after a long NEDA period. However, it could provide some support to physicians in advising patients, who do no longer want to continue DMT because of sustained freedom of disease activity. In this context, neurologists should encourage younger people to continue DMT despite no overt clinical or MRI indicators of ongoing disease activity, while in patients $>45$ years, stopping DMT together with continuous regular clinical and MRI follow-up may provide a clinically reasonable option with low risk.

Funding Open access funding provided by Medical University of Vienna.

\section{Compliance with ethical standard}

Conflicts of interests PA: participated in meetings sponsored by, received speaker honoraria or travel funding from Biogen, Merck, Roche, Sanofi-Genzyme and Teva. He received honoraria for consulting with Biogen and a combined research sponsorship from Biogen, Merck, Sanofi-Genzyme, Roche, and Teva for the development of a smartphone application for people with MS. He received directed funding from Quanterix International for research studying serum neurofilaments as a biomarker in MS. Thomas Berger: has participated in meetings sponsored by and received honoraria (lectures, advisory boards, consultations) from pharmaceutical companies marketing treatments for multiple sclerosis: Almirall, Biogen, Bionorica, Celgene, MedDay, Merck, Novartis, Roche, Sanofi Aventis/Genzyme, TG Therapeutics and TEVA. His institution has received financial support in the last 2 years by unrestricted research grants (Biogen, Novartis, Sanofi Aventis/Genzyme, Roche, TEVA) and for participation in clinical trials in multiple sclerosis sponsored by Alexion, Bayer, Biogen, Merck, Novartis, Roche, Sanofi Aventis/Genzyme, TEVA. Gabriel Bsteh: has participated in meetings sponsored by, received speaker honoraria or travel funding from Biogen, Celgene, Merck, Novartis, Sanofi-Genzyme and Teva, and received honoraria for consulting Biogen, Roche and Teva. Assunta D-B: has participated in meetings sponsored by or received speaker honoraria or travel funding or unrestricted scientific grants from Biogen, Merck, Novartis and Roche. FL: has participated in meetings sponsored by, received speaker honoraria or travel funding or unrestricted scientific grants from Actelion, Biogen, Celgene, Med Day, Merck, Novartis, Roche, Sanofi-Genzyme, Schering, and Teva. 
TM: has participated in meetings sponsored by or received travel funding from Biogen, Celgene, Merck, Novartis, Roche, Sanofi-Genzyme and Teva. Paulus Stefan Rommer: received honoraria for lectures or consultancy from AbbVie, Alexion, Allmiral, Biogen, Daiichi-Sankyo, Merck, Novartis, Roche, Sandoz, Sanofi Genzyme, Teva. He received research grants from Amicus, Biogen, Merck, Roche. Sabine S-P: declares no conflict of interest with respect to the study and data presented in this paper. TZ: has participated in meetings sponsored by or received travel funding from Biogen, Merck, Novartis, Roche, SanofiGenzyme and Teva.

Ethical standard statement The study was approved by the ethics committee of the Medical University of Vienna (EK 1203/2016) and have therefore been performed in accordance with the ethical standards laid down in the 1964 Declaration of Helsinki and its later amendments.

Informed consent Due to retrospective data collection, no informed consent was obtained.

Open Access This article is licensed under a Creative Commons Attribution 4.0 International License, which permits use, sharing, adaptation, distribution and reproduction in any medium or format, as long as you give appropriate credit to the original author(s) and the source, provide a link to the Creative Commons licence, and indicate if changes were made. The images or other third party material in this article are included in the article's Creative Commons licence, unless indicated otherwise in a credit line to the material. If material is not included in the article's Creative Commons licence and your intended use is not permitted by statutory regulation or exceeds the permitted use, you will need to obtain permission directly from the copyright holder. To view a copy of this licence, visit http://creativecommons.org/licenses/by/4.0/.

\section{References}

1. McDonald WI, Compston A, Edan G et al (2001) Recommended diagnostic criteria for multiple sclerosis: Guidelines from the International Panel on the Diagnosis of Multiple Sclerosis. Ann Neurol. https://doi.org/10.1002/ana.1032

2. Confavreux C, Vukusic S, Adeleine P (2003) Early clinical predictors and progression of irreversible disability in multiple sclerosis: An amnesic process. Brain. https://doi.org/10.1093/brain/awg081

3. Confavreux C, Vukusic S (2006) Natural history of multiple sclerosis: A unifying concept. Brain. https://doi.org/10.1093/brain/ awl007

4. Dobson R, Topping J, Davis A et al (2013) Cerebrospinal fluid and urinary biomarkers in multiple sclerosis. Acta Neurol Scand. https://doi.org/10.1111/ane.12119

5. Fisniku LK, Brex PA, Altmann DR et al (2008) Disability and T2 MRI lesions: A 20-year follow-up of patients with relapse onset of multiple sclerosis. Brain. https://doi.org/10.1093/brain/awm32 9

6. Thompson AJ, Banwell BL, Barkhof F et al (2018) Diagnosis of multiple sclerosis: 2017 revisions of the McDonald criteria. Lancet Neurol 17:162-173

7. Jacobs LD, Beck RW, Simon JH et al (2000) Intramuscular interferon beta-1a therapy initiated during a first demyelinating event in multiple sclerosis. N Engl J Med. https://doi.org/10.1056/ NEJM200009283431301

8. Kappos L, Polman CH, Freedman MS et al (2006) Treatment with interferon beta- $1 \mathrm{~b}$ delays conversion to clinically definite and McDonald MS in patients with clinically isolated syndromes. Neurology. https://doi.org/10.1212/01.wnl.0000237641.33768.8d
9. Comi G, Martinelli V, Rodegher M et al (2009) Effect of glatiramer acetate on conversion to clinically definite multiple sclerosis in patients with clinically isolated syndrome (PreCISe study): a randomised, double-blind, placebo-controlled trial. Lancet. https ://doi.org/10.1016/S0140-6736(09)61259-9

10. Comi G, De Stefano N, Freedman MS et al (2012) Comparison of two dosing frequencies of subcutaneous interferon beta$1 \mathrm{a}$ in patients with a first clinical demyelinating event suggestive of multiple sclerosis (REFLEX): A phase 3 randomised controlled trial. Lancet Neurol. https://doi.org/10.1016/S1474 -4422(11)70262-9

11. Salhofer-Polanyi S, Cetin H, Leutmezer F et al (2017) Epidemiology of Multiple Sclerosis in Austria. Neuroepidemiology. https:// doi.org/10.1159/000479696

12. Bsteh G, Feige J, Ehling R et al (2017) Discontinuation of diseasemodifying therapies in multiple sclerosis - Clinical outcome and prognostic factors. Mult Scler. https://doi.org/10.1177/13524 58516675751

13. Kister I (2017) Disease-modifying therapies can be safely discontinued in an individual with stable relapsing-remitting MS - YES. Mult Scler. https://doi.org/10.1177/1352458517709957

14. Tremlett H, Zhao Y, Devonshire V (2008) Natural history of secondary-progressive multiple sclerosis. Mult Scler. https://doi. org/10.1177/1352458507084264

15. Kister I, Spelman T, Alroughani R et al (2016) Discontinuing disease-modifying therapy in MS after a prolonged relapse-free period: A propensity score-matched study. J Neurol Neurosurg Psychiatry. https://doi.org/10.1136/jnnp-2016-313760

16. Kister I, Spelman T, Patti F et al (2018) Predictors of relapse and disability progression in MS patients who discontinue disease-modifying therapy. J Neurol Sci. https://doi.org/10.1016/j. jns.2018.06.001

17. Yano H, Gonzalez C, Healy BC et al (2019) Discontinuation of disease-modifying therapy for patients with relapsing-remitting multiple sclerosis: Effect on clinical and MRI outcomes. Mult Scler Relat Disord. https://doi.org/10.1016/j.msard.2019.07.021

18. Barkhof F, Filippi M, Miller DH et al (1997) Comparison of MRI criteria at first presentation to predict conversion to clinically definite multiple sclerosis. Brain. https://doi.org/10.1093/brain $/ 120.11 .2059$

19. Hegen H, Bsteh G, Berger T (2018) No evidence of disease activity' - is it an appropriate surrogate in multiple sclerosis? Eur $\mathbf{J}$ Neurol 25:1107-e101

20. Kappos L, Edan G, Freedman MS et al (2016) The 11-year longterm follow-up study from the randomized BENEFIT CIS trial. Neurology. https://doi.org/10.1212/WNL.0000000000003078

21. Galetta SL (2001) The Controlled High Risk Avonex ${ }^{\circledR}$ Multiple Sclerosis trial (CHAMPS STUDY). J Neuro-Ophthalmol 21:292-295

22. Tremlett H, Zhao Y, Joseph J et al (2008) Relapses in multiple sclerosis are age- and time-dependent. J Neurol Neurosurg Psychiatry. https://doi.org/10.1136/jnnp.2008.145805

23. Tutuncu M, Tang J, Zeid NA et al (2013) Onset of progressive phase is an age-dependent clinical milestone in multiple sclerosis. Mult Scler J. https://doi.org/10.1177/1352458512451510

24. Tobin WO, Weinshenker BG (2017) Disease-modifying therapies can be safely discontinued in an individual with stable relapsingremitting MS - NO. Mult Scler. https://doi.org/10.1177/13524 58517702556

25. Bsteh G, Hegen H, Dosser C et al (2020) To treat or not to treat: Sequential individualized treatment evaluation in relapsing multiple sclerosis. Mult Scler Relat Disord. https://doi.org/10.1016/j. msard.2019.101908 